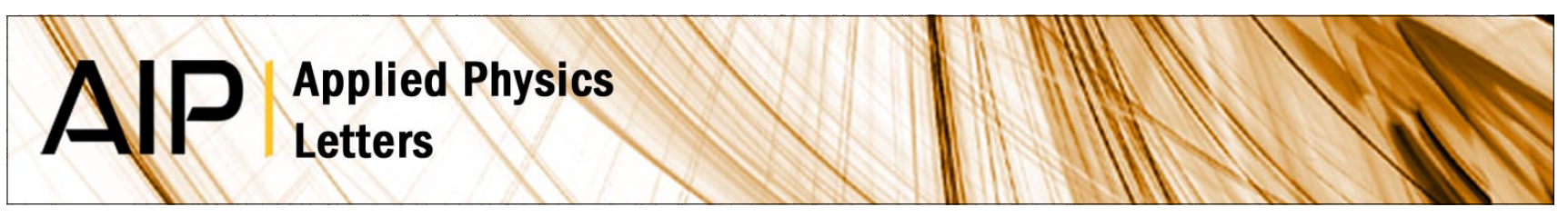

\title{
Magnetic domain wall pinning by a curved conduit
}

E. R. Lewis, D. Petit, L. Thevenard, A. V. Jausovec, L. O'Brien et al.

Citation: Appl. Phys. Lett. 95, 152505 (2009); doi: 10.1063/1.3246154

View online: http://dx.doi.org/10.1063/1.3246154

View Table of Contents: http://apl.aip.org/resource/1/APPLAB/v95/i15

Published by the American Institute of Physics.

\section{Related Articles}

Modeling plastic deformation effect on magnetization in ferromagnetic materials J. Appl. Phys. 111, 063909 (2012)

Micromagnetic analysis of switching and domain structure in amorphous metallic nanowires Appl. Phys. Lett. 100, 122404 (2012)

Microstructure dependence of Barkhausen voltage pulse width in steel J. Appl. Phys. 111, 063903 (2012)

Characterization of domain wall-based traps for magnetic beads separation J. Appl. Phys. 111, 07B336 (2012)

Current-driven domain wall motion in heterostructured ferromagnetic nanowires Appl. Phys. Lett. 100, 112401 (2012)

\section{Additional information on Appl. Phys. Lett.}

Journal Homepage: http://apl.aip.org/

Journal Information: http://apl.aip.org/about/about_the_journal

Top downloads: http://apl.aip.org/features/most_downloaded

Information for Authors: http://apl.aip.org/authors

\section{ADVERTISEMENT}

\section{(@) ACCELERATE AMBER AND NAMD BY 5X. NVIDIA TRYIT ONA FREE, REMOTELYYHOSTED CLUSTER.}




\title{
Magnetic domain wall pinning by a curved conduit
}

\author{
E. R. Lewis, ${ }^{\text {a) }}$ D. Petit, L. Thevenard, A. V. Jausovec, L. O’Brien, D. E. Read, and \\ R. P. Cowburn \\ Department of Physics, Blackett Laboratory, Imperial College London, Prince Consort Road, \\ London SW7 2BW, United Kingdom
}

(Received 4 August 2009; accepted 20 September 2009; published online 13 October 2009)

\begin{abstract}
The pinning of a magnetic domain wall in a curved Permalloy (NiFe) nanostrip is experimentally studied. We examine the dependence of the pinning on both the radius of curvature of the bend and the chirality of the transverse domain wall. We find that bends act as potential wells or potential barriers depending on the chirality of the domain wall; the pinning field in both cases increases with decreasing radius of curvature. Micromagnetic simulations are consistent with the experimental results and show that both exchange and demagnetizing energies play an important role. (C) 2009 American Institute of Physics. [doi:10.1063/1.3246154]
\end{abstract}

The behavior of magnetic domain walls (DWs) in thin ferromagnetic strips has been widely studied in recent years, driven by the promise of applications in magnetic logic and memory devices. ${ }^{1,2}$ Such devices may require curves in the nanostrip, with sharper curvature needed for higher storage densities; in addition, numerous experiments involve DW interactions in structures with varying curvature. For example, several studies examine the magnetization states of ring structures with varying curvature such as ellipses, ${ }^{3}$ squares, ${ }^{4}$ or triangles ${ }^{5}$ other experiments use curved sections of wire to locally create DWs. ${ }^{6-9}$ However, the physics governing the effect of the curvature on the DW has not yet been considered in detail. In this paper, we systematically study the interaction of transverse DWs with bends, varying both the radius of curvature of the bend and the chirality of the DW, which is defined by the sense of rotation of magnetic moments crossing the DW. ${ }^{10}$ We find that bends act either as potential wells or potential barriers, depending on the DW chirality; the pinning field in both cases increases with decreasing radius of curvature.

Two contributions to DW pinning by a bend may be expected. The first is an effective geometrical pinning due to the deviation of the strip from the field direction, which means that the projection of the field onto the direction of DW motion is reduced. This effect is independent of the curvature and of the DW chirality. The second contribution is intrinsic pinning due to a change in the potential landscape seen by the DW when the curvature changes. It is this effect which we are interested in studying. In addition to the curvature, the intrinsic pinning may be affected by the DW chirality; chirality-dependent pinning has previously been observed, for example, at constrictions and protrusions in a nanostrip. ${ }^{7,11}$ For our nanostrip dimensions, the stable DW structure is a transverse $\mathrm{DW}^{12,13}$ with a characteristic triangular shape. The orientation of the triangle depends on the DW chirality. For example, Fig. 1(a) shows a simulated head-to-head ( $\mathrm{HH})$ DW with core magnetization pointing upwards and the wide side of the DW on the upper edge of the nanostrip; a DW of the same chirality, i.e., tail-to-tail (TT) with core magnetization pointing downwards, also has its wide side on the upper edge of the nanostrip. We describe the

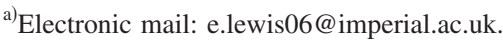

DW-bend configuration as "wide" when the wide side of the DW is on the outside of the bend, or "narrow" when the narrow side of the DW is on the outside of the bend.

In order to study these effects, Permalloy $\left(\mathrm{Ni}_{81} \mathrm{Fe}_{19}\right)$ nanostrips $100 \mathrm{~nm}$ wide and $10 \mathrm{~nm}$ thick were fabricated on $\mathrm{Si}$ substrates using electron beam lithography, thermal evaporation and a lift-off process. For magnetic characterization, the sample was placed at the center of a quadrupole electromagnet, allowing fields to be applied in any direction in the sample plane. Magnetization switching of single nanostructures was measured using a high-sensitivity magnetooptical Kerr effect (MOKE) magnetometer in a longitudinal geometry, with a focused laser spot diameter of $\sim 5 \mu \mathrm{m}$.

Schematics of the two types of structure used are shown in Figs. 1(b) and 1(c). The left-hand end of each structure is patterned into an $\mathrm{L}$ or $\mathrm{C}$ shape with outer radius of curvature $500 \mathrm{~nm}$ (large enough to give negligible intrinsic pinningsee later). A $90^{\circ}$ bend of outer radius $R$ is patterned at the

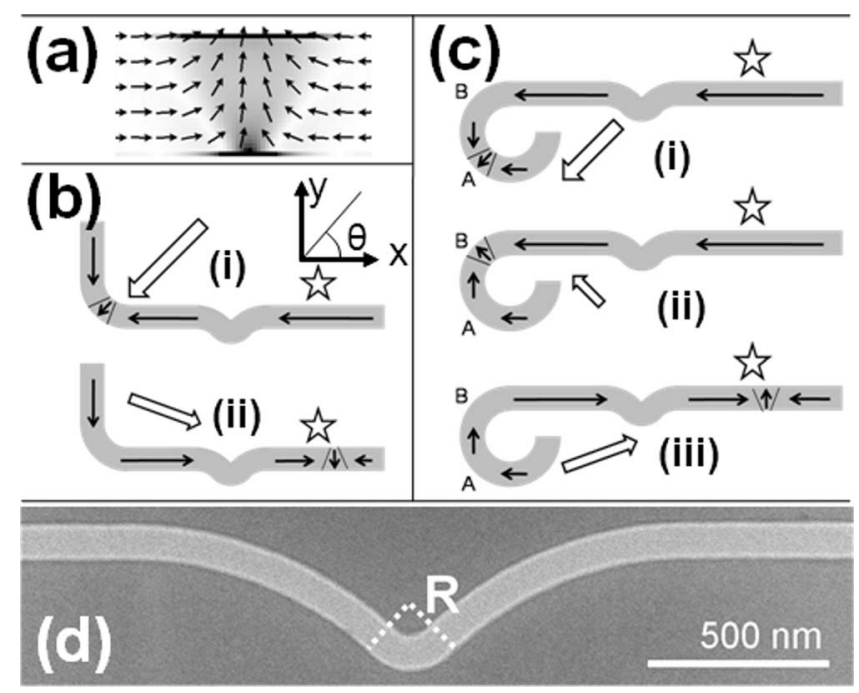

FIG. 1. (a) Micromagnetic simulation of transverse DW structure. The arrows indicate the direction of the magnetization, $\mathbf{M}$, and the grayscale indicates $|\nabla \cdot \mathbf{M}|$, where $\nabla \cdot \mathbf{M}=0$ is colored white. [(b) and (c)] schematics of $L$ and $\mathrm{C}$ shaped structures and first half of field sequences used for transmission field measurements. The position of the MOKE laser spot is indicated by the star. (d) SEM image of middle portion of a typical nanostructure with central bend of radius $R=149 \mathrm{~nm}$. 
center of the horizontal arm, flanked by two $45^{\circ}$ sections with outer radius $1050 \mathrm{~nm}$. A scanning electron microscopy (SEM) image of the central portion of one nanostructure is shown in Fig. 1(d). $R$ was varied between 42 and $500 \mathrm{~nm}$; for $R<100 \mathrm{~nm}$, proximity effects in the lithography process cause a small increase in width $(\sim 15 \mathrm{~nm}$ for the smallest $R)$ at the center of the bend, with an inner radius of $\sim 20 \mathrm{~nm}$. The $\mathrm{L}$ and $\mathrm{C}$ shaped ends allow DWs to be created with either orientation relative to the bend: L shapes [Fig. 1(b)] give the wide configuration, and $\mathrm{C}$ shapes [Fig. 1(c)] the narrow configuration. We first measured the "transmission field," $H_{T}$, required to move a DW through the central bend. The field sequences used are shown in Figs. 1(b) and 1(c). The duration of each sequence is $1 \mathrm{~s}$. A HH DW is created at the left-hand end of the structure by applying a saturating field of magnitude $\sim 460$ Oe along the direction $(-1,-1)$. For the L-shaped structures, the field is then reduced at $45^{\circ}$ to $H_{x}=0, H_{y}=-18 \mathrm{Oe}$, followed by a gradual increase of $H_{x}$ to +300 Oe. The sequence is then repeated with all fields reversed, so that a TT DW is initially created. The MOKE spot is positioned on the right-hand side of the structure and magnetization switching is observed when $H_{x}=H_{T}$. Measurements on the left-hand side showed that the DW depins from the left-hand corner at $15 \pm 3$ Oe. The C-shaped structures are initialized with the same saturating field along $(-1,-1)$, and the field is reduced at $45^{\circ}$ to $H_{x}=-19, H_{y}=0$ Oe. The field direction then rotates to bring the DW from position A to position $\mathrm{B}$, at $H_{x}=0, H_{y}=17 \mathrm{Oe} ; H_{x}$ is then increased to $300 \mathrm{Oe}$ as before and magnetization switching is again measured on the right-hand part of the structure. The sequence is then repeated with all fields reversed. Note that the DW could also have been created at position B; however, creating the DW at position A ensures that the central bend is initialized in the same way for both structures. We also checked that DWs are not created at the central bend: we reversed the $\mathrm{x}$-component of the field sequence shown in Fig. 1(b), so that the initial saturating field is applied along $(1,-1)$ and no DW is created at the left-hand end. We found that on both sides of the bend, switching only occurred at the nucleation field, $H_{N}=213 \pm 14$ Oe: no DWs are observed to propagate out of the central bend, showing that DWs are either not created at the central bend, or two DWs are created but immediately annihilate when the field is reversed.

The measured $\left|H_{T}\right|$, averaged over HH and TT DWs, is plotted for both configurations as a function of $R$ in Fig. 2(a), where each data point corresponds to a separate structure. The error in each measurement depends on the width of the step in the MOKE signal, which has an average value of $6 \pm 3$ Oe; the symbol height on the graphs is 10 Oe. Each measurement is averaged over 150 cycles of the magnetic field. It can be seen that $H_{T}$ decreases with increasing $R$, and is higher for the narrow configuration. The dependence on $R$ and on the DW chirality becomes weak for $R>350 \mathrm{~nm}$. In the narrow configuration, $H_{T}$ saturates for very small $R$ at the nucleation field $H_{N}=213 \pm 14$ Oe (upper dashed line); for large $R$ both configurations saturate at the effective

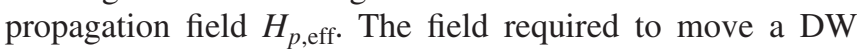
along a $10 \mu \mathrm{m}$ horizontal strip has been measured as $H_{p}$ $=12 \pm 2$ Oe. The projection of the field along a strip oriented at an angle $\theta$ is $H_{\text {tangent }}=H_{x} \cos \theta+H_{y} \sin \theta$; for our structures, $\theta_{\max }= \pm 45^{\circ} . H_{p \text {,eff }}$ is the value of $H_{x}$ for which the minimum $H_{\text {tangent }}$ is equal to $H_{p}$. The calculated value of
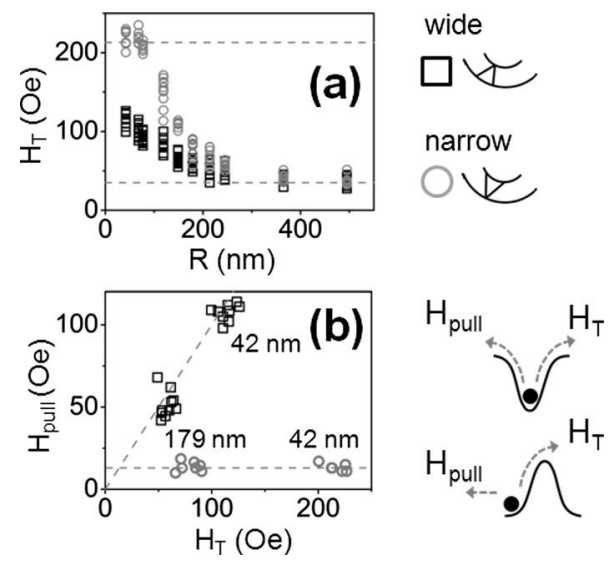

FIG. 2. Transmission and potential measurements for wide (squares) and narrow (circles) configurations (see schematics at top right). (a) Measured transmission fields, $H_{T}$, as a function of $R$. The dashed lines indicate the nucleation field $H_{N}=213 \pm 14$ Oe and the effective propagation field $H_{p \text {, eff }}$ $=35 \pm 6$ Oe. (b) $H_{\text {pull }}$ as a function of $H_{T}$ for structures with $R=42 \mathrm{~nm}$ and $R=179 \mathrm{~nm}$. The dashed lines indicate $H_{\text {pull }}=H_{T}$ and $H_{\text {pull }}=H_{p}=12 \pm 2 \mathrm{Oe}$. The schematics describe the relation between $H_{\text {pull }}$ and $H_{T}$ for a DW trapped inside a potential well (top) and a DW blocked by a potential barrier (bottom)

$H_{p \text {,eff }}$ is $35 \pm 6$ Oe, indicated by the lower dashed line on Fig. 2(a).

One possible complication could be transformation of the DW structure from one chirality to another during its motion, ${ }^{14}$ which we have previously observed in nanostrips of very similar dimensions. ${ }^{15,16}$ In Ref. 15 we observed that around $60 \%$ of structures did not show evidence of DW transformations. In the remaining $40 \%$, the DW was sometimes transformed and sometimes not. We might then expect to see a systematic difference between the nominal wide and narrow configurations, but with a minority of measured switching fields corresponding to the opposite configuration. However, we see little evidence of this: the data for the wide and narrow configurations are well separated. This apparent absence of DW transformations may be due to increased roughness; ${ }^{17}$ alternatively, the measurements may not have been sensitive enough to detect such transformations, since they were averaged over half as many cycles of the magnetic field.

We performed a second type of measurement to determine the potential landscape (e.g., potential well or barrier) experienced by the DW. The DW is created in the same way as for the $H_{T}$ measurements, and pushed toward the bend with a maximum field $H_{\text {push }}$; the field is then reversed to pull the DW back from the central bend. Because the value of $H_{\text {push }}$ may not be enough to sweep the DW through the bend and out of the structure, the final part of the sequence applies a large rotating field to sweep the DW out of the left-hand side of the structure; this ensures that the initial magnetization configuration is always set in the same way. The MOKE spot is now positioned on the left-hand part of the structure, so that switching is measured when the DW moves out of the left-hand corner and again when the field reaches $H_{\text {pull, }}$, the value required to bring the DW back from the central bend; however, if $H_{\text {push }}>H_{T}$, the DW passes through the central bend and annihilates at the end of the wire, so that the measured $H_{\text {pull }}=H_{N}$. We found that for each structure, all values of $H_{\text {push }}<H_{T}$ give the same value of $H_{\text {pull }}$. This value of $H_{\text {pull }}$ is plotted for structures in both configurations with $R$ 


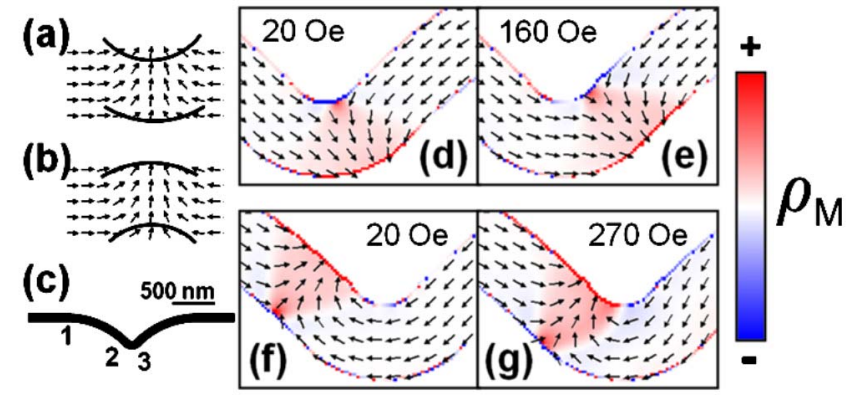

FIG. 3. (Color online) [(a) and (b)] Curving the nanostrip edges into the narrow configuration (a) tends to increase the perpendicular component of $\mathbf{M}$ and create surface charges; the converse is true for the wide configuration (b). [(c)-(g)] Micromagnetic simulations of magnetization configurations for $R=150 \mathrm{~nm}$. (c) Simulated bend structure. [(d)-(g)] Magnetization configurations for the wide $[(\mathrm{d})$ and $(\mathrm{e})]$ and narrow $[(\mathrm{f})$ and $(\mathrm{g})]$ cases. Each picture shows the stable configuration at the field indicated. The arrows and color indicate $\mathbf{M}$ and $\rho_{M}=-\mu_{0} \nabla \cdot \mathbf{M}$, respectively, as shown in the color scale at right.

$=42 \mathrm{~nm}$ and $R=179 \mathrm{~nm}$ as a function of $H_{T}$ in Fig. 2(b). It can be seen that in the wide case, $H_{\text {pull }} \sim H_{T}$; once the DW reaches the bend, the same field is required either to transmit the DW, or to bring it back to the starting position: the bend acts as a potential well. In the narrow case, $H_{\text {pull }} \sim$ constant $=H_{p}$, indicating that the bend acts as a potential barrier. Until the DW is pushed over the barrier, it can be brought back to the starting position with a small field independent of the barrier height. These behaviors are illustrated in the schematics in Fig. 2(b).

Because the Py nanostrips have negligible magnetocrystalline anisotropy, the pinning by a bend must be due to changes in the exchange energy, $E_{\text {ex }}$, and demagnetizing energy, $E_{\mathrm{dem}}$, of the DW when it is positioned on a curved portion of the nanostrip. The sign of the change in $E_{\mathrm{ex}}$ is intuitive because the spins rotate through a smaller total angle in the wide case [Fig. 3(e)] than on a straight strip, and a larger total angle in the narrow case [Fig. 3(g)], giving a decrease or increase respectively in the total $E_{\mathrm{ex}}$, consistent with the experimentally observed well or barrier. $E_{\text {dem }}$ has a complex dependence on the DW structure; recent work ${ }^{18}$ has shown, for example, that the complete distribution of the magnetization, $\mathbf{M}$, must be taken into account for an accurate calculation of the interaction between DWs even when they are in separate nanostrips. However, we can make qualitative arguments using similar reasoning to that in Ref. 7 for DW pinning at constrictions and protrusions. The spins crossing the DW turn out of the easy direction and acquire a component perpendicular to the strip edge. In the narrow configuration, the strip edges tend to lie perpendicular to the local $\mathbf{M}$ within the DW, which will tend to create surface charges and therefore increase the demagnetizing energy; the converse is true in the wide case, where the strip edges tend to align with the local M. This is described schematically in Figs. 3(a) and 3(b).

We used the OOMMF package ${ }^{19}$ to simulate the DW-bend interaction for a structure with $R=150 \mathrm{~nm}$ [Fig. 3(c)]. The magnetization configurations are shown in Figs. 3(d) $-3(\mathrm{~g})$; the color indicates the divergence or "magnetic charge density" $\rho_{M}=-\mu_{0} \nabla \cdot \mathbf{M}$. This provides an alternative picture of the DW shape, which can be difficult to visualize directly from the distribution of M. Under a field $H_{x}=10$ Oe (not shown), the DW is pinned at position 1 [Fig. 3(c)] due to edge roughness introduced by the pixelation. At $20 \mathrm{Oe}$, the DW has moved toward the bend: in the wide case, the DW sits inside the bend (potential well) at position 3 [Fig. 3(d)]; in the narrow case, the DW sits before the bend (potential barrier) at position 2 [Fig. 3(f)]. Pictures (e) and (g) show the configurations just before transmission. The discrepancy between simulated and experimental transmission fields is attributed to temperature effects as discussed in e.g., Ref. 7. The calculated energy changes are found to be on the order of a few $\mathrm{eV}$ and are consistent with the experimentally observed well or barrier: when the DW first moves toward the bend, both $E_{\mathrm{ex}}$ and $E_{\mathrm{dem}}$ are found to decrease in the wide configuration as the DW falls into the potential well, but change very little in the narrow configuration, where the DW remains blocked in front of the barrier. As the field increases, $E_{\mathrm{ex}}$ and $E_{\mathrm{dem}}$ increase in both cases as the DW is pushed out of the well or against the barrier. The two energy terms change by comparable amounts, showing that both effects play an important role in the pinning.

In conclusion, we have experimentally studied the interaction of transverse DWs with bends, where we vary both the radius of curvature of the bend and the orientation of the transverse DW with respect to the bend. We find that bends act as potential wells when the wide side of the DW is on the outside of the bend, and potential barriers in the opposite case. The pinning field in both cases increases with decreasing radius of curvature. Micromagnetic simulations are consistent with the experimental results and show that both exchange and demagnetizing energies play an important role.

${ }^{1}$ D. A. Allwood, G. Xiong, C. C. Faulkner, D. Atkinson, D. Petit, and R. P. Cowburn, Science 309, 1688 (2005).

${ }^{2}$ S. S. P. Parkin, M. Hayashi, and L. Thomas, Science 320, 190 (2008).

${ }^{3}$ F. J. Castaño, C. A. Ross, and A. Eilez, J. Phys. D 36, 2031 (2003).

${ }^{4}$ P. Vavassori, M. Grimsditch, V. Novosad, V. Metlushko, and B. Ilic, Phys. Rev. B 67, 134429 (2003).

${ }^{5}$ P. Vavassori, D. Bisero, V. Bonanni, A. Busato, M. Grimsditch, K. M. Lebecki, V. Metlushko, and B. Ilic, Phys. Rev. B 78, 174403 (2008).

${ }^{6}$ A. Vanhaverbeke, A. Bischof, and R. Allenspach, Phys. Rev. Lett. 101, 107202 (2008).

${ }^{7}$ D. Petit, A. V. Jausovec, D. Read, and R. P. Cowburn, J. Appl. Phys. 103, 114307 (2008).

${ }^{8}$ G. Nahrwold, L. Bocklage, J. M. Scholtyssek, T. Matsuyama, B. Kruger, U. Merkt, and G. Meier, J. Appl. Phys. 105, 07d511 (2009).

${ }^{9}$ C. W. Sandweg, N. Wiese, D. McGrouther, S. J. Hermsdoerfer, H. Schultheiss, B. Leven, S. McVitie, B. Hillebrands, and J. N. Chapman, J. Appl. Phys. 103, 093906 (2008).

${ }^{10}$ Note that strictly speaking a chirality cannot be defined for a transverse DW on a straight strip; the system can only be assigned a "handedness" when some other asymmetry is introduced, such as a bend.

${ }^{11}$ M. Hayashi, L. Thomas, C. Rettner, R. Moriya, X. Jiang, and S. S. P. Parkin, Phys. Rev. Lett. 97, 207205 (2006).

${ }^{12}$ R. D. McMichael and M. J. Donahue, IEEE Trans. Magn. 33, 4167 (1997).

${ }^{13}$ Y. Nakatani, A. Thiaville, and J. Miltat, J. Magn. Magn. Mater. 290, 750 (2005).

${ }^{14}$ N. L. Schryer and L. R. Walker, J. Appl. Phys. 45, 5406 (1974).

${ }^{15}$ E. R. Lewis, D. Petit, A. V. Jausovec, L. O’Brien, D. E. Read, H. T. Zeng, and R. P. Cowburn, Phys. Rev. Lett. 102, 057209 (2009).

${ }^{16}$ Transformations were previously found to occur over distances of less than $2 \mu \mathrm{m}$; in the present experiment the DW travels more than $10 \mu \mathrm{m}$.

${ }^{17}$ Y. Nakatani, A. Thiaville, and J. Miltat, Nature Mater. 2, 521 (2003).

${ }^{18}$ L. O’Brien, D. Petit, H. T. Zeng, E. R. Lewis, J. Sampaio, A. V. Jausovec, D. E. Read, and R. P. Cowburn, Phys. Rev. Lett. 103, 077206 (2009).

${ }^{19}$ The OOMMF code is available at http://math.nist.gov/oommf/. 